\title{
Tidal Variations of the Earth Rotation
}

\author{
J. M. Ferrándiz \\ Dept. Matemática Aplicada, University of Alicante, Spain
}

Yu. V. Barkin

Sternberg Astronomical Institute, Moscow, Russia

J. Getino

Faculty of Sciences, University of Valladolid, Spain

\begin{abstract}
The equations for the rotation of a weakly deformable celestial body in non canonical Andoyer variables have been used to study the perturbation of Earth rotation due to tidal deformation raised by the Moon and Sun. A theory of the perturbed rotational motion of an isolated weakly deformable body in Andoyer variables and in components of the angular velocity has been developed. Mantle tidal deformations due to lunar and solar influences were analytically described and taken into account. Perturbations of the first order in the Earth's polar motion were determined.
\end{abstract}

\section{Equations of a Weakly Deformable Body Rotation}

Consider a weakly deformable body, assuming that its particles are slightly displaced from the positions corresponding to a rigid motion, or move with small velocity that is a known function of time. The body has an inner rigid part, to which we tie a Cartesian reference system $C \xi \eta \zeta$, and an external deformable envelope. The origin of both of them coincides with the center of mass of the body.

Let $A, B, C$ and $F, E, D$ be axial and centrifugal moments of inertia of the body with respect to the said frame, that are assumed to be known functions of time. The components of the inertia tensor can be cast as a sum of the terms:

$$
\begin{array}{lll}
A=A_{0}+\delta A, & B=B_{0}+\delta B, & C=C_{0}+\delta C, \\
F=F_{0}+\delta F, & E=E_{0}+\delta E, & D=D_{0}+\delta D,
\end{array}
$$

where $A_{0}, B_{0}, C_{0}, F_{0}, E_{0}, D_{0}$ are the components of the tensor of inertia in the undeformed state of the body, and $\delta A, \delta B, \delta C, \delta F, \delta E, \delta D$ are additional terms due to the density redistribution. To focus our attention on the effects pointed out before, we will suppose that the angular momentum of the relative motion of the body particles vanishes (i.e., we choose a Tisserand frame). 
Now we introduce for consideration a set of Andoyer's variables

$$
G, \theta, \rho, l, g, h,
$$

with $\cos \theta=L / G, \cos \rho=H / G$ in the usual notation.

A full description of these variables is given in the papers by Kinoshita (1977), Getino and Ferrándiz (1990), Kubo (1991), and Barkin (1999). In the last papers the differential equations of rotational motion of a weakly deformable body in the Andoyer variables were obtained in the general case.

In this paper we will study the influence of the deformations of the Earth's mantle on its rotation. Tidal variations of the components of the inertia tensor of the Earth were given in Ferrándiz and Getino (1993). We will start with the simplified case in which the Earth is an isolated, weakly deformable elastic body, and consider the set of equations of motion:

$$
\begin{aligned}
\frac{d \theta}{d t}= & G \sin \theta\left\{\frac{\delta F}{A_{0} B_{0}} \cos 2 l\right\}+\frac{G}{C_{0} A_{0}} \cos \theta(\delta E \cos l-\delta D \sin l), \\
\frac{d l}{d t}= & G \cos \theta\left\{\frac{1}{C_{0}}-\frac{\delta C}{C_{0}}-\frac{1}{A_{0}}-\frac{\delta A}{A_{0}^{2}}-\frac{\delta F}{A_{0}^{2}} \sin 2 l\right\}- \\
& -\frac{G}{C_{0} A_{0}} \cos e c \theta \cos 2 \theta(\delta E \sin l-\delta D \cos l), \\
\frac{d g}{d t}= & G\left(\frac{1}{A_{0}}-\frac{\delta A}{A_{0}^{2}}-\frac{\delta F}{A_{0}^{2}} \sin 2 l\right)+\frac{G}{C_{0} A_{0}} \cot \theta(\delta E \sin l+\delta D \cos l),
\end{aligned}
$$

obtained from equations (5) by Ferrándiz and Barkin (this proceedings) for the case of an axisymmetric body $\left(A_{0}=B_{0}\right)$. In this case three first integrals exist: $G=G_{0}, \rho=\rho_{0}, h=h_{0}$. This implies the angular momentum vector of the rotational motion of this deformable body is constant. $G_{0}, \rho_{0}$ and $h_{0}$ are initial values of the corresponding Andoyer variables. Equations (3) are basic to determining the perturbations in the Earth's rotation. Perturbations of the components of the angular velocity are defined by using the transformation formulae from Andoyer variables to the tidal variation $(\delta p, \delta q, \delta r)$ of the angular velocity:

$$
\begin{aligned}
\delta p= & -\frac{G \delta A}{A_{0}^{2}} \sin \delta \sin l-\frac{\delta P}{A_{0}}+\frac{\delta F}{A_{0} B_{0}} G \sin \theta \cos l+\frac{\delta E}{A_{0} C_{0}} G \cos \theta+ \\
& +\frac{G}{A_{0}} \cos \theta \sin l \delta \theta+\frac{G}{A_{0}} \sin \theta \cos l \delta l, \\
\delta q= & -\frac{G \delta B}{B_{0}^{2}} \sin \delta \cos l-\frac{\delta Q}{B_{0}}+\frac{\delta F}{A_{0} B_{0}} G \sin \theta \sin l+\frac{\delta D}{B_{0} C_{0}} G \cos \theta+ \\
& +\frac{G}{B_{0}} \cos \theta \cos l \delta \theta+\frac{G}{B_{0}} \sin \theta \sin l \delta l, \\
\delta r= & -\frac{G \delta C}{C_{0}^{2}} \cos \delta-\frac{\delta R}{C_{0}}+\frac{\delta E}{A_{0} C_{0}} G \sin \theta \sin l+\frac{\delta D}{B_{0} C_{0}} G \sin \theta \cos l- \\
& -\frac{G}{C_{0}} \sin \theta \delta \theta .
\end{aligned}
$$




\section{Model of the Earth mantle tidal deformation}

For the determination of the variations in the Earth's polar motion caused by lunisolar tidal deformations of the mantle, we use tidal variations of the secondharmonic coefficients, presented in the form of the following series:

$$
\begin{aligned}
\delta J_{2} & =\sum_{\sigma} K_{2}(\sigma) \cos \Theta_{\sigma}, \\
\delta C_{22} & =\sum_{\sigma} K_{22 a}(\sigma) \cos \left(2 l+2 g-\Theta_{\sigma}\right)+\sum_{\sigma} K_{22 b}(\sigma) \cos \left(2 l+2 g+\Theta_{\sigma}\right), \\
\delta S_{22} & =-\sum_{\sigma} K_{22 a}(\sigma) \sin \left(2 l+2 g-\Theta_{\sigma}\right)-\sum_{\sigma} K_{22 b}(\sigma) \sin \left(2 l+2 g+\Theta_{\sigma}\right),(5) \\
\delta C_{21} & =\sum_{\sigma} K_{21 a}(\sigma) \sin \left(l+g-\Theta_{\sigma}\right)+\sum_{\sigma} K_{21 b}(\sigma) \sin \left(l+g+\Theta_{\sigma}\right), \\
\delta S_{21} & =\sum_{\sigma} K_{21 a}(\sigma) \cos \left(l+g-\Theta_{\sigma}\right)+\sum_{\sigma} K_{21 b}(\sigma) \cos \left(l+g+\Theta_{\sigma}\right),
\end{aligned}
$$

where $K_{22 a}(\sigma), \ldots, K_{21 b}(\sigma)$ are numerical coefficients, the values of which are given in Table 1 of Ferrándiz and Getino (1993). The argument $\Theta_{\sigma}$ is a integer linear combination of the angles of the lunar theory:

$$
\begin{aligned}
\Theta_{\sigma} & =\sigma_{1} l_{M}+\sigma_{2} l_{S}+\sigma_{3} F+\sigma_{4} D+\sigma_{5} \Omega, \\
F & =l_{M}+g_{M}, \quad D=l_{M}+g_{M}+h_{M}-l_{S}-g_{S}-h_{S}, \\
\sigma & =\left(\sigma_{1}, \sigma_{2}, \sigma_{3}, \sigma_{4}, \sigma_{5}\right),
\end{aligned}
$$

Here $l_{M}, g_{M}, h_{M}$ and $l_{S}, g_{S}, h_{S}$ are the Delaunay variables for the Moon and the Sun and $S=l+g$ is the angle of the Earth's rotation (referred to the $O_{\xi}$ axis).

Variations of the components of the Earth's inertia tensor are connected with variations $(4,5)$ by simple relationships and can be presented in the following way.

$$
\begin{aligned}
\frac{\delta A}{C} & =\sum_{\sigma}\left\{A_{\sigma} \cos \Theta_{\sigma}+A_{\sigma,-2} \cos \left(-2 S+\Theta_{\sigma}\right)+A_{\sigma, 2} \cos \left(\Theta_{\sigma}+2 S\right)\right\} \\
\frac{\delta B}{C} & =\sum_{\sigma}\left\{B_{\sigma} \cos \Theta_{\sigma}+B_{\sigma,-2} \cos \left(\Theta_{\sigma}-2 S\right)+B_{\sigma, 2} \cos \left(\Theta_{\sigma}+2 S\right)\right\} \\
\frac{\delta C}{C} & =\sum_{\sigma}\left\{C_{\sigma} \cos \Theta_{\sigma}\right\} \\
\frac{\delta F}{C} & =\sum_{\sigma}\left\{F_{\sigma, 2}^{*} \sin \left(\Theta_{\sigma}+2 S\right)+F_{\sigma,-2}^{*} \sin \left(\Theta_{\sigma}-2 S\right)\right\} \\
\frac{\delta E}{C} & =\sum_{\sigma}\left\{E_{\sigma, 1}^{*} \sin \left(\Theta_{\sigma}+S\right)+E_{\sigma,-1}^{*} \sin \left(\Theta_{\sigma}-S\right)\right\} \\
\frac{\delta D}{C} & =\sum_{\sigma}\left\{D_{\sigma, 1} \cos \left(\Theta_{\sigma}+S\right)+D_{\sigma,-1} \cos \left(\Theta_{\sigma}-S\right)\right\} .
\end{aligned}
$$

The coefficients of the series (5) and (7) are connected by simple relations:

$$
A_{\sigma}=B_{\sigma}=-\frac{1}{3 J} K_{2}(\sigma), C_{\sigma}=-2 A_{\sigma},
$$




$$
\begin{aligned}
A_{\sigma, 2} & =-B_{\sigma, 2}=F_{\sigma, 2}^{*}=\frac{2}{J} K_{22 b}(\sigma) \\
A_{\sigma,-2} & =-B_{\sigma, 2}=F_{\sigma,-2}^{*}=\frac{2}{J} K_{22 a}(\sigma) \\
E_{\sigma, 1}^{*} & =D_{\sigma, 1}=-\frac{1}{J} K_{21 b}(\sigma), \quad E_{\sigma,-1}^{*}=D_{\sigma,-1}=-\frac{1}{J} K_{21 a}(\sigma)
\end{aligned}
$$

Numerical values of coefficients (8) are displayed in Table 1.

\begin{tabular}{|c|c|c|c|c|c|c|c|c|c|c|c|}
\hline $\mathrm{N}$ & $l$ & $l^{\prime}$ & $F$ & $\mathrm{D}$ & $\Omega$ & $\begin{array}{l}A_{\sigma, 0} \\
=B_{\sigma}\end{array}$ & $\begin{aligned} & A_{\sigma, 2} \\
= & F_{\sigma, 2}^{\bullet} \\
= & B_{\sigma, 2}\end{aligned}$ & $\begin{aligned} & A_{\sigma,-2} \\
= & F_{\sigma,-2} \\
= & B_{\sigma_{1}-2}\end{aligned}$ & $\begin{array}{l}C_{\sigma, 0}= \\
-2 A_{\sigma, 0}\end{array}$ & $\begin{array}{c}A_{\sigma, 1}^{\bullet}= \\
D_{\sigma, 1}^{\prime}\end{array}$ & $\begin{array}{c}E_{\sigma,-1}^{\bullet}= \\
D_{\sigma_{1}-1}^{*}\end{array}$ \\
\hline 1. & 1 & 0 & 0 & -2 & 0 & -0.2049 & -0.0321 & -0.0321 & 0.4098 & 0.1470 & 0.1470 \\
\hline 2. & 1 & 0 & 0 & 0 & 0 & -1.0716 & -0.1669 & -0.1669 & 2.1433 & 0.7690 & 0.7690 \\
\hline 3. & 0 & 0 & 0 & 2 & 0 & -0.1778 & -0.0278 & -0.0278 & 0.3556 & 0.1276 & 0.1276 \\
\hline 4. & 1 & 0 & 2 & 0 & 1 & -0.1610 & -0.0073 & 0.1681 & 0.3220 & 0.0517 & -0.3529 \\
\hline 5. & 0 & 0 & 2 & 0 & 1 & -0.8409 & -0.0381 & 0.8788 & 1.6818 & 0.2695 & -1.8444 \\
\hline 6. & 0 & 0 & 0 & 0 & 1 & 0.8501 & 0.0381 & -0.8885 & -1.7001 & -0.2725 & 1.8650 \\
\hline 7. & -1 & 0 & 2 & 2 & 2 & -0.0738 & -0.0018 & -0.8570 & 0.1476 & 0.0154 & -0.3556 \\
\hline 8. & -1 & 0 & 2 & 0 & 2 & 0.0574 & 0.0012 & 0.6665 & -0.1147 & -0.0118 & 0.2764 \\
\hline 9. & 1 & 0 & 2 & 0 & 2 & -0.3885 & -0.0085 & -4.5131 & 0.7770 & 0.0804 & -1.8725 \\
\hline 10. & $\hat{0}$ & 0 & 2 & 2 & 2 & -0.0620 & -0.0012 & -0.7209 & 0.1240 & 0.0130 & -0.2991 \\
\hline 11. & 0 & 0 & 2 & 0 & 2 & -2.0286 & -0.0436 & -23.5732 & 4.0571 & 0.4207 & -9.7796 \\
\hline 12. & 0 & 1 & 0 & 0 & 0 & -0.1499 & -0.0236 & -0.0236 & 0.2998 & 0.1077 & 0.1077 \\
\hline 13. & 0 & 1 & 2 & -2 & 2 & -0.0552 & -0.0012 & -0.6411 & 0.1105 & -0.0027 & -0.2661 \\
\hline 14. & 0 & 0 & 2 & -2 & 2 & -0.9417 & -0.0206 & -10.9411 & 1.8834 & -0.0484 & -4.5394 \\
\hline
\end{tabular}

Table 1. Coefficients of the main periodic tidal variations of the axial and centrifugal moments of inertia of the Earth (unit $=10^{-9} \mathrm{rad}$ ).

\section{Variations of the Earth's polar motion due to lunisolar tidal vari- ations of its mantle}

The solution to the rotation of an isolated and deformable body was first obtained in the Andoyer variables. First order perturbations of the angular velocity components were also derived, taking into account results on the unperturbed Chandler motion of an axisymmetric weakly deformable body (Barkin, in press) and the transformation formulae (4). Here we use the solution of the problem in variables $p, q, r$.

Tidal variations of the moments of inertia of the Earth lead to the following perturbations of the pole motion:

$$
\begin{aligned}
\delta p & =\sum_{\sigma} p_{\sigma, 1}^{*} \sin \left(\Theta_{\sigma}+S\right)+p_{\sigma,-1}^{*} \sin \left(\Theta_{\sigma}-S\right) \\
\delta q & =\sum_{\sigma} q_{\sigma, 1} \cos \left(\Theta_{\sigma}+S\right)+q_{\sigma,-1} \cos \left(\Theta_{\sigma}-S\right) \\
p_{\sigma, \varepsilon}^{*} / \omega & =q_{\sigma, \varepsilon} / \omega \frac{E_{\sigma}^{*}}{C_{0}}\left[1+\frac{\omega \Omega+\left(\Omega_{\sigma}+\varepsilon \omega\right)}{\left(\Omega_{\sigma}+\varepsilon \omega\right)^{2}-\Omega^{2}}\right]
\end{aligned}
$$

where $\Omega$ is the Chandler frequency, and $\omega$ is the Earth's angular velocity. 
Using formulae (9) we find the corresponding coefficients of the tidal lunisolar variations in the polar motion of the Earth (Table 2). Similar perturbations in the diurnal rotation of the Earth were described in detail earlier (Yoder et al. 1981, Ferrándiz and Getino, 1993).

Amplitudes of perturbations of the equatorial angular velocity components are given in Table 2 .

Table 2. Coefficients of the periodic perturbations in the pole motion of the Earth caused by its tidal deformation (unit $=0$ ".001).

\begin{tabular}{rrrrrrrr}
$\mathrm{N}$ & $l_{M}$ & $l_{S}^{\prime}$ & $\mathrm{F}$ & $\mathrm{D}$ & $\Omega$ & $p_{\sigma, 1}^{*} / \omega=q_{\sigma, 1} / \omega$ & $p_{\sigma,-1}^{*} / \omega=q_{\sigma,-1} / \omega$ \\
\hline 1. & 1 & 0 & 0 & -2 & 0 & 0.062 & -0.001 \\
2. & 1 & 0 & 0 & 0 & 0 & 0.311 & -0.006 \\
3. & 0 & 0 & 0 & 2 & 0 & 0.051 & -0.028 \\
4. & 1 & 0 & 2 & 0 & 1 & 0.098 & 0.009 \\
5. & 0 & 0 & 2 & 0 & 1 & 0.107 & 0.031 \\
6. & 0 & 0 & 0 & 0 & 1 & -0.056 & -0.001 \\
7. & -1 & 0 & 2 & 2 & 2 & 0.003 & 0.009 \\
8. & -1 & 0 & 2 & 0 & 2 & -0.005 & -0.002 \\
9. & 1 & 0 & 2 & 0 & 2 & 0.015 & 0.049 \\
10. & 0 & 0 & 2 & 2 & 2 & 0.005 & 0.010 \\
11. & 0 & 0 & 2 & 0 & 2 & 0.081 & 0.165 \\
12. & 0 & 1 & 0 & 0 & 0 & 0.0443 & -0.000 \\
13. & 0 & 1 & 2 & -2 & 2 & -0.001 & 0.001 \\
14. & 0 & 0 & 2 & -2 & 2 & -0.020 & 0.007
\end{tabular}

\section{Summary}

The tidal perturbations in the Earth's polar motion were tabulated for the Ferrándiz and Getino model of the mantle deformations using theoretical results on perturbed rotational motion of a deformable body by Getino and Ferrándiz (1999) and Barkin (in press).

Acknowledgments. JMF and JG's work has been partially supported by Spanish Projects ESP97-1816-C04-02 (CICYT) and PB95-696 (DGES). Yu.B's stay at University of Alicante has been partially funded by grant INV99-01-06 (Generalitat Valenciana).

\section{References}

Barkin, Yu.V. (in press), Astronomical and Astrophysical Transactions.

Ferrándiz, J.M. \& Getino, J. 1993, Celestial Mechanics and Dynamical Astronomy, 57, 279-292.

Ferrándiz, J.M. \& Barkin, Yu. V. This proceedings.

Getino, J. \& Ferrándiz, J.M. 1990 Celestial Mechanics, 49, 303-326.

Getino, J. \& Ferrándiz, J.M. 1991 Celestial Mechanics, 52, 381-396.

Kinoshita, H. 1977 Celestial Mechanics, 15, 277-326.

Kubo, Y. 1991 Celestial Mechanics, 50, 165-187.

Yoder, C.F. et al. 1981 J. Geophys. Res., 86, 881-891. 